\title{
A New Multiphase Soft Segmentation with Adaptive Variants
}

\author{
Hongyuan Wang, ${ }^{1}$ Fuhua Chen, ${ }^{2}$ and Yunmei Chen ${ }^{3}$ \\ ${ }^{1}$ School of Information Science \& Engineering, Changzhou University, Changzhou 213164, China \\ ${ }^{2}$ Department of Natural Science \& Mathematics, West Liberty University, West Liberty, WV 26074, USA \\ ${ }^{3}$ Department of Mathematics, University of Florida, Gainesville, FL 36011, USA
}

Correspondence should be addressed to Fuhua Chen; fuhua.chen@westliberty.edu

Received 19 February 2013; Accepted 9 May 2013

Academic Editor: Zhang Yi

Copyright (C) 2013 Hongyuan Wang et al. This is an open access article distributed under the Creative Commons Attribution License, which permits unrestricted use, distribution, and reproduction in any medium, provided the original work is properly cited.

\begin{abstract}
Soft segmentation is more flexible than hard segmentation. But the membership functions are usually sensitive to noise. In this paper, we propose a multiphase soft segmentation model for nearly piecewise constant images based on stochastic principle, where pixel intensities are modeled as random variables with mixed Gaussian distribution. The novelty of this paper lies in three aspects. First, unlike some existing models where the mean of each phase is modeled as a constant and the variances for different phases are assumed to be the same, the mean for each phase in the Gaussian distribution in this paper is modeled as a product of a constant and a bias field, and different phases are assumed to have different variances, which makes the model more flexible. Second, we develop a bidirection projected primal dual hybrid gradient (PDHG) algorithm for iterations of membership functions. Third, we also develop a novel algorithm for explicitly computing the projection from $R^{K}$ to simplex $\Delta_{K-1}$ for any dimension $K$ using dual theory, which is more efficient in both coding and implementation than existing projection methods.
\end{abstract}

\section{Introduction}

Image segmentation has played an important role in image processing and computer vision. Recently, variational segmentation models have attracted increasing interest [1-8]. With level set technique [9], variational models can be solved efficiently. The technique was originally developed for two-phase segmentation, and then extended to multiphase segmentation [10-13]. With carefully choosing the initial values, these methods can achieve an ideal solution for multiphase segmentation. However, the nonconvexity of the energy functional in the level set formulation is an inherent drawback of level set-method. As a result, many level setbased variational segmentation models are sensitive to initial values, especially for multiphase segmentation, and may lead to an inferior local minimum.

One way to overcome the drawback is to use fuzzy membership function to replace the Heaviside function of a level set function in level set formulation for modeling a characteristic function, called soft segmentation. With this relaxation (characteristic function can be viewed as a special case of membership function), Chan and Bresson et al. [1, 2, 5] proved that global minimum can be achieved in these models due to the convexity of the energy functional. Unfortunately, this method cannot be easily applied to multi-phase segmentation. Very recently, based on a variational convexification technique developed by Pock et al. [14], Brown et al. [15], and Bae et al. [16] provide different ways to find globally optimal solution for piecewise constant Mumford-Shah model (more generally called continuous Potts model).

There have been many soft segmentation methods [1722]. Mory and Ardon extended the original region competition model [8] to a fuzzy region competition method $[19,20]$. The technique generalizes some existing supervised and unsupervised region-based models. The proposed functional is convex, which guarantees the global solution in the supervised case. Unfortunately, this method only applies to two-phase segmentation and is hard to be extended to multiphase segmentation. Fuzzy C-mean (FCM) is a method developed for pattern classification and recognition and has been applied to image segmentation $[17,18,21]$. The standard FCM model partitions a data set $\left\{x_{k}\right\}_{k=1}^{N} \subset R^{d}$ into $M$ clusters 
by the following objective function:

$$
J_{\mathrm{FCM}}=\sum_{i=1}^{N} \sum_{k=1}^{M} u_{i k}\left\|x_{i}-v_{k}\right\|_{2}^{2}
$$

where $u_{i k} \geq 0$ is the membership value of datum $x_{i}$ for class $k$ with $\sum_{k=1}^{M} u_{i k}=1$ and $v_{k}$ stands for the cluster centers [23, 24]. The original FCM method is very sensitive to noise. Pham et al. proposed an adaptive fuzzy C-mean (AFCM) model [21] which is more robust to noise than the standard FCM, where the constant cluster centers $v_{k}$ used in the FCM model (1) are substituted by functions that are smooth enough and close to the corresponding cluster centers.

Another class of soft segmentations is based on stochastic approaches [18, 22, 25]. In these approaches, pixel intensities are considered as samples of one or several random variables. The advantage of stochastic method is its stronger ability to deal with random noise. In most stochastic segmentation models, the likelihood functions are used to represent the fitting term in an energy functional. It starts from the assumption that reasonable segmentation should maximize the likelihood. The method is called maximum likelihood (ML) method [26]. An expectation-maximization (EM) algorithm is usually employed to solve it when data is incomplete. However, simply using likelihood to model an image is not enough since it ignored the prior knowledge of an image. In [18], a segmentation framework based on maximum a posteriori principle (MAP) was proposed for partial volume (PV) segmentation of MRI brain images, which is a classic application of soft segmentation.

Shen proposed a general multiphase stochastic variational fuzzy segmentation model combining stochastic principle and Modica-Mortola's phase-transition theory [22]. The intensity of images was modeled as a mixed Gaussian distribution. The model assumed that membership functions should be either close to 1 or close to 0 , which simplified the model but limited its application. For example, it is not reasonable to apply the model to partial volume segmentation since in that case the membership functions are usually neither close to 1 nor close to 0 at the boundary of different matters.

Bias correction is an important mean in soft segmentation to deal with intensity inhomogeneity [21, 27-29]. For example, Wells et al. proposed an expectation-maximization (EM) algorithm to solve the bias correction problem and the tissue classification problem [29]. The EM algorithm was used to iteratively estimate the posterior tissue class probabilities when the bias field is known and to estimate the MAP of the bias field when tissue class probabilities are known. The disadvantage of this method is that the directly computed bias field may not be smooth which will lead to a poor bias correction and segmentation results. Pham and Prince proposed an adaptive fuzzy C-means algorithm which is formulated by modifying the objective function in the fuzzy C-means algorithm to include a multiplicative bias field, which allows the centroids of each class to vary across the image. Smoothness of the bias field is ensured by penalizing its first and second order derivatives, which leads to a computationally expensive procedure for the smoothing of the bias field. Ahmed et al. proposed to add a neighborhood term that enabled the class membership of a pixel to be influenced by its neighbors [27]. The neighborhood term acts as a regularizer and forces the solution toward a piecewise homogeneous labeling. Li et al. proposed a variational level set-based method for medical image segmentation and bias correction [28]; the smoothness of the bias field is intrinsically ensured by the data term in the variational formulation.

In all the above papers related to Gaussian distributions, there is a common feature in that all assume that the variants for different phases are same. However, when the variants of different phases are quite different (It is true sometime. Experiments are also provided later.), the models introduced above may misclassify the phases. In this paper, we propose a stochastic variational model for multiphase soft segmentation in the presence of noise and intensity inhomogeneity, where the image intensity at each point is modeled as a mixed Gaussian distribution with means and variances to be optimized. Different from Shen's work [22], our model does not set the assumption that membership functions must be close to either 1 or 0 . So, our model is more suitable for soft segmentation and application to partial volume analysis. Since our model is developed based on the assumption that the image intensity is a mixed Gaussian distribution with possibly different variances for different phases, it is also different from $[18,21]$ in that our model adaptively corrects bias of intensities and removes noise by finding optimized mean and adaptive variances. It is demonstrated by experiments that our model is not only robust to noise but also powerful in bias correction. The model can be implemented very fast using a bidirection projected PDHG algorithm. The rest of the paper is organized as follows. The new model is developed in Section 2. The numerical implementation scheme is presented in Section 3. In Section 4, we show some experiment results and also give some explanation and analysis. Both synthetic images and authentic images are used. Finally, we summarize the paper with a short conclusion.

\section{Model Development}

Let $I(x)$ be a $2 \mathrm{D}$ image defined on an open bounded domain $\Omega$ containing $K$ phases. Let $w$ be phase label variable (i.e., $w(x) \in\{1, \ldots, K\}$ for all $x \in \Omega)$. At each pixel $x$, both $w(x)$ and $I(x)$ are viewed as random variables indexed by $x$. The probability that $x$ belongs to the $i$ th phase is represented by the ownership functions $p_{i}(x), 1 \leq i \leq K$. If we denote the probability density function (PDF) of the random variable $I(x)$ given that $x$ belongs to the $i$ th phase by $\operatorname{Prob}(I(x)$ | $w(x)=i)$, then the PDF of $I(x)$ is a mixed distribution given by

$$
\sum_{i=1}^{K} \operatorname{Prob}(I(x) \mid w(x)=i) p_{i}(x) .
$$

Suppose in further that $\operatorname{Prob}(I(x) \quad \mid w(x)=i)$ is a Gaussian PDF for each $i=1, \ldots, K$ and all random variables 
$\{I(x): x \in \Omega\}$ are independent. Then the likelihood (joint $\mathrm{PDF})$ is

$$
\prod_{x \in \Omega} \sum_{i=1}^{K} g\left(I \mid u_{i}(x), \sigma_{i}\right) p_{i}(x)
$$

where

$$
g(x \mid \mu, \sigma)=\frac{1}{(\sqrt{2 \pi} \sigma)} \exp \left(-\frac{(x-\mu)^{2}}{2 \sigma^{2}}\right) .
$$

The negative log-likelihood is

$$
E[I \mid P, U, \sigma]=-\int_{\Omega} \log \left(\sum_{i=1}^{K} g\left(I \mid u_{i}(x), \sigma_{i}\right) p_{i}(x)\right),
$$

where $P=\left[p_{1}, p_{2}, \ldots, p_{K}\right], U=\left[u_{1}, u_{2}, \ldots, u_{K}\right]$, and $\sigma=$ $\left[\sigma_{1}, \sigma_{2} \ldots, \sigma_{K}\right]$. When each $u_{i}(x)$ is chosen to be a constant $c_{i}$, respectively, and $\sigma_{i}=\sigma$ for all $i(i=1,2, \ldots, K)$, where $\sigma$ is a fixed constant, then the model is deduced to a piecewise constant model. In paper $[18,21], u_{i}(x)$ is constructed as the product of a constant $c_{i}$ and a full field bias function $b(x)$ which is assumed to be close to 1 . Now we assume $u_{i}(x)=$ $c_{i} b(x)$ in (5) and let $c=\left(c_{1}, c_{2}, \ldots, c_{K}\right)$, which leads to the following energy functional $E_{F}(p, b, c, \sigma)$ :

$$
\begin{aligned}
& E_{F}(p, b, c, \sigma) \\
& =\lambda \int_{\Omega}-\log \left[\sum_{i=1}^{K} \frac{1}{\sqrt{2 \pi} \sigma_{i}} \exp \left(-\frac{\left(I(x)-b(x) c_{i}\right)^{2}}{2 \sigma_{i}^{2}}\right) p_{i}\right] d x
\end{aligned}
$$

By adding the $L^{2}$-norm of $\nabla b$ and the total variation of $p_{i}(x)$ to $E_{F}(p, b, c, \sigma)$ as regularity terms for bias field $b(x)$ and membership functions $p_{i}(x)$, respectively, we get the following energy functional $E_{F R}(p, b, c, \sigma)$ with Gaussian mixture and bias correction:

$$
\begin{aligned}
& E_{F R}(p, b, c, \sigma) \\
& =\lambda \int_{\Omega}-\log \left[\sum_{i=1}^{K} \frac{1}{\sqrt{2 \pi} \sigma_{i}} \exp \left(-\frac{\left(I(x)-b(x) c_{i}\right)^{2}}{2 \sigma_{i}^{2}}\right) p_{i}\right] d x \\
& \quad+\mu \int_{\Omega}|\nabla b|^{2} d x+\sum_{i=1}^{K} \int_{\Omega}\left|\nabla p_{i}\right| d x \\
& \triangleq-\lambda \int_{\Omega} \log \left(\sum_{i=1}^{K} f_{i}(x) p_{i}(x)\right) d x+\mu \int_{\Omega}|\nabla b|^{2} d x \\
& \quad+\sum_{i=1}^{K} \int_{\Omega}\left|\nabla p_{i}\right| d x .
\end{aligned}
$$

Remark. We want to mention that our model is not the first time to use Gaussian distribution. On the contrary, the Gaussian distribution has been introduced to many segmentation models, such as graph cut [30] and soft Mumford-Shah model
[22]. The difference between the proposed model and the previous models is that those previous models all assume that different Gaussian distributions have a same variance and are usually fixed. However, in our model, we assume that different Gaussian distributions have different variances which increase the flexibility.

\section{Numerical Implementation}

Note that the energy functional is convex with respect to all its variables except for variances. For fixed variances, global minimization can be achieved for any initialization. The Euler-Lagrange equations of variances, means, and bias are as follows:

$$
\begin{gathered}
-\lambda \int_{\Omega} \frac{f_{i} p_{i}\left(\left(I-b c_{i}\right)^{2}-\sigma_{i}^{2}\right)}{2 \sigma_{i}^{4} \sum_{i=1}^{K} f_{i} p_{i}} d x=0, \\
-\frac{\lambda}{\sigma_{i}^{2}} \int_{\Omega} \frac{f_{i} p_{i}\left(I-b c_{i}\right) b}{\sum f_{i} p_{i}} d x=0, \\
-\lambda \frac{f_{i} p_{i}}{\sum_{i=1}^{K} f_{i} p_{i}} \frac{\left(I-b c_{i}\right) c_{i}}{\sigma_{i}^{2}}-\Delta b=0 .
\end{gathered}
$$

Correspondingly, we use the following iteration schemes:

$$
\begin{gathered}
\sigma_{i}^{(n+1)}=\sigma_{i}^{(n)}+t_{\sigma} \lambda\left[\int_{\Omega} \frac{f_{i} p_{i}\left(\left(I-b c_{i}\right)^{2}-\sigma_{i}^{2}\right)}{2 \sigma_{i}^{4} \sum_{i=1}^{K} f_{i} p_{i}} d x\right]^{(n)}, \\
c_{i}^{(n+1)}=c_{i}^{(n)}+t_{c}\left[\frac{\lambda}{\sigma_{i}^{2}} \int_{\Omega} \frac{f_{i} p_{i}\left(I-b c_{i}\right) b}{\sum f_{i} p_{i}} d x\right]^{(n)}, \\
b^{(n+1)}=b^{(n)}+t_{b}\left[\lambda \frac{f_{i} p_{i}}{\sum_{i=1}^{K} f_{i} p_{i}} \frac{\left(I-b c_{i}\right) c_{i}}{\sigma_{i}^{2}}+\Delta b\right]^{(n)} .
\end{gathered}
$$

The challenge in the implementation is the optimization of membership functions $p_{i}(x)$ because of the constraints

$$
1 \geq p_{i}(x) \geq 0, \quad \sum_{i=1}^{K} p_{i}(x)=1
$$

which requires $p=\left(p_{1}, p_{2}, \ldots, p_{K}\right)$ to be in the simplex $\Delta_{K-1}$. There have been two ways to deal with the simplex constraint. One is to use Lagrangian multiplier method (or augmented Lagrangian multiplier method) for $\sum_{i=1}^{K} p_{i}(x)=$ 1 , and add an exact penalty term for each $0 \leq p_{i}(x) \leq 1$ (see $[1,5,31])$. The drawback of Lagrangian multiplier method is its low convergence rate. The so-called exact penalty term is exact only under some constraint and is not differentiable at end points and must be replaced by a smoothed version for approximation which finally hurts the exactness. Another way to deal with the simplex constraint is to use the EulerLagrangian equation of the unconstraint problem for iterations and then project each iteration result to the simplex $\Delta_{K-1}$ [22]. The drawback of this method is that no general analytic expression can be written for all dimensions. For 
different dimensions, the projection functions are different and need to be written in a different way. Particularly, when the dimension is greater than three, the projection function becomes complicated, which leads to a low efficiency in both coding and implementation. In this paper, we give a novel way of projection using dual method. The projection can be expressed uniformly for all dimensions, and the analytic property is guaranteed due to dual theory.

Dual method has been extensively studied to deal with total variation which is not differentiable at points where the first-order variation is zero. One of the popular example is Chambolle dual method [32]. Recently, Zhu and Chan developed a new algorithm combining the gradient decent method and dual method, called primal dual hybrid gradient method (PDHG) (see [33] for details). The method integrates the advantages of both gradient method and dual method, and thus is faster than using either method. It is proved to be faster than using dual method only, and its modified iteration form is guaranteed to converge when step size satisfies some condition (see [34-36]). In our application, we adopted the ideal of PDHG and apply it to our model with constraint on simplex $\Delta_{K-1}$.

3.1. Optimize Membership Functions Using PDHG. By the principle of PDHG, to minimize (7) with respect to membership functions $p_{i}(i=1, \ldots, K)$ under constraint (12), it is equivalent to solve the following discrete min-max problem:

$$
\max _{q \in X^{K}} \min _{p \in \Delta_{K-1}}-\langle p, D q\rangle-H(p),
$$

where $p=\left(p_{1}, \ldots, p_{K}\right), q=\left(q_{1}, \ldots, q_{K}\right)$, and

$$
\begin{aligned}
H(p) & =\lambda \sum_{i, j=1}^{m, n} \log \langle f(i, j), p(i, j)\rangle \\
& =\lambda \sum_{i, j=1}^{m, n} \log \sum_{k=1}^{K} f_{k}(i, j) p_{k}(i, j) .
\end{aligned}
$$

The descent direction for $\min _{p \in R^{K}}\langle p, D q\rangle+H(p)$ is $D q+$ $\nabla_{p} H(p)$. So, the evolution of membership $p$ (primal step) is

$$
p^{(n+1)}=P_{\Delta_{K-1}}\left(p^{(n)}+\tau_{n}\left(D q^{(n)}+\nabla H\left(p^{(n)}\right)\right)\right),
$$

where $P_{\Delta_{K-1}}(v)$ is the projection to the simplex defined by

$$
P_{\Delta_{K-1}}(x) \triangleq \min _{z \in \Delta_{K-1}}\|z-x\|
$$

for $\forall x \in R^{K}$, where $\|\cdot\|$ denotes the Euclidean distance. We will see a novelty method for the projection to simplex in the next section.

Since the first variation of (13) with respect to $q_{i}$ is $D p_{i}$, the dual step is

$$
q_{i}^{(n+1)}=P_{X^{K}}\left(q_{i}^{(n)}+\theta^{(n)} D p_{i}^{(n)}\right),
$$

where $P_{X^{K}}$ is the projection to space $T$ defined by

$$
\left[P_{X^{K}}(x)\right]_{l}=\frac{x_{l}}{\max \left\{\|x\|_{2}, 1\right\}},
$$

where $l$ denotes the number of component of a vector.
Therefore, the bi-direction projected PDHG algorithm for minimizing energy functional ( 7 ) is given by

$$
\begin{gathered}
p^{(n+1)}=P_{\Delta_{K-1}}\left(p^{(n)}+\tau_{n}\left(D q^{(n)}+\nabla H\left(p^{(n)}\right)\right)\right), \\
q^{(n+1)}=P_{X^{K}}\left(q^{(n)}+\lambda \nabla p^{(n)}\right),
\end{gathered}
$$

where the projection to simplex $\Delta_{K-1}$ is given explicitly in the next section.

3.2. Projection to Simplex $\Delta_{K-1}$. Now, we want to deduce the explicit solution for $P_{\Delta_{K-1}}(x)$. Let $x^{*}$ denote the solution. Define

$$
\Pi_{\Delta_{K-1}}(x)= \begin{cases}0 & \text { if } x \in \Delta_{K-1} \\ \infty & \text { otherwise }\end{cases}
$$

Then by Morean's Identity, we have

$$
\begin{aligned}
x^{*} & =P_{\Delta_{K-1}}(x) \triangleq \min _{z \in \Delta_{K-1}}\|z-x\| \\
& =\underset{x \in R^{K}}{\arg \min }\left\{\frac{1}{2}\|x-y\|^{2}+\Pi_{\Delta_{K-1}}(x)\right\} \\
& =y-\underset{z \in R^{K}}{\arg \min }\left\{\Pi_{\Delta_{K-1}}^{*}(z)+\frac{1}{2}\|z-y\|^{2}\right\},
\end{aligned}
$$

where $\Pi_{\Delta_{K-1}}^{*}(z)$ is the Legendre-Fenchel transform of $\Pi_{\Delta_{K-1}}$. By definition, we have

$$
\begin{aligned}
\Pi_{\Delta_{K-1}}^{*}(z) & =\sup _{x \in R^{K}}\left\{\langle x, z\rangle-\Pi_{\Delta_{K-1}}(x)\right\} \\
& =\sup _{x \in \Delta_{K-1}}\langle x, z\rangle \\
& =\max _{1 \leq i \leq K} z_{i} \triangleq t .
\end{aligned}
$$

So, we finally get the solution

$$
x_{i}^{*}=y-z_{i}^{*}=\max \left(y_{i}-t, 0\right), \quad i=1,2, \ldots, K
$$

which is simple and very fast.

3.3. Summary of the Algorithm. In this section, we display the complete algorithm as below.

Algorithm 1.

(1) Initialization:

(a) Initialize means $c=\left[c_{1}, c_{2}, \ldots, c_{n}\right]$;

(b) Initialize variants $\sigma=\left[\sigma_{1}, \sigma_{2}, \ldots, \sigma_{n}\right]$;

(c) Initialize memberships $p=\left[p_{1}(x), p_{2}(x)\right.$, $\left.\ldots, p_{n}(x)\right]$ with $p_{i}(x) \in[0,1]$

(d) Initialize bias $b=\left[b_{1}(x), b_{2}(x), \ldots, b_{n}(x)\right]$ with $b_{i}(x) \in[-1,1]$

(e) Initialize parameters $\lambda$ and $\mu$. 

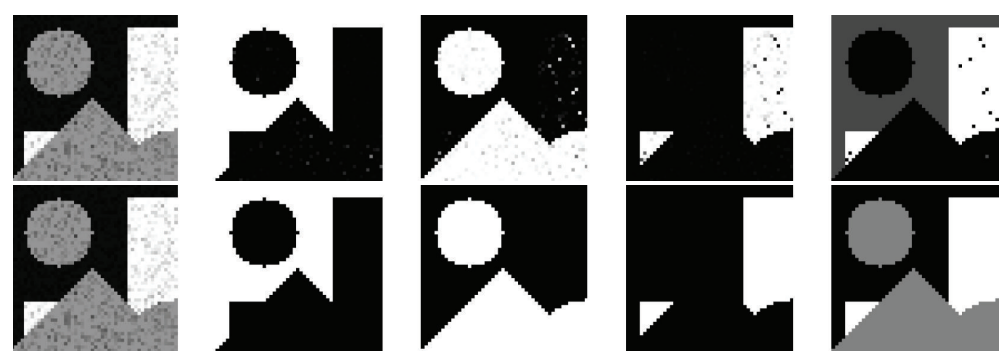

FIGURE 1: Experiment 1: robustness to noise. The first line: segmentation using Shen's model. The second line: segmentation using the proposed model. For each line, from left to right are original image, three membership functions, and hard segmentation after thresholding.

(2) Iterations:

(a) Update variances with (9);

(b) Update means with (10);

(c) Update bias with (11);

(d) Update memberships (three times):

(i) Initialize $p_{i}$ and $q_{i}$;

(ii) Initialize $\tau$;

(iii) Update $p$ and $q$ with (19).

(3) Termination: The iteration will be terminated if the changes of means are all less than a pre-set threshold $\epsilon>0$.

The choice of parameters $\lambda$ and $\mu$ depends on specific images. Usually, at the beginning, $\lambda$ should be chosen big enough. After some iterations, decreasing $\lambda$ can accelerate the convergence. The parameter $\tau$ must be $\leq 2 / 9$ to guarantee the convergence [32]. The efficiency of the algorithm PDHG can be found from [37].

\section{Experiment and Discussion}

Since the main difference between our model and other Gaussian distribution-based model lies in the variable variants, we especially show the difference between variants varied and variants fixed. Since our model can be viewed as an extension of Shen's paper [22], we present many experimental results based on a comparison with Shen's model.

The first experiment aims at testing robustness to noise. In Figure 1, the original image contains obviously three phases. We added a mixed Gaussian noise with zero mean and an overall variance 0.03 . First, we applied Shen's model. We choose $\lambda_{1}=5$ and stop iterations using criterion $\max _{1 \leq i \leq 3}\left\{\left|c(i)_{\text {new }}-c(i)_{\text {old }}\right|\right\}<0.001$, where $c(i)_{\text {old }}$ denotes the old mean before each iteration and $c(i)_{\text {new }}$ denotes the new mean after each iteration (the same for the rest experiments). Then we applied our model (7) to the image. Obviously, the result of the new model is much better.

Explanation Analysis. This big difference comes from the difference of the fitting terms in two models. Note that in Shen's model, to make the fitting term small enough, the image intensity at each point must be very close to the mean of its phase. Thus it is sensitive to noise. Comparatively, in model
(7), the effect of isolated noise to the energy functional can be counteracted by the variances appeared in the denominators of the fitting term. So the new model is more robust to noise.

The second experiment aims at comparing robustness to bias. In Figure 2, the first line is the original biased image and its ground truth of all three membership functions. The second line and the third line show the soft segmentations obtained using Shen's model and the proposed model, respectively. Obviously, the proposed model gives more precise result compared with the ground truth since there is no bias in the segmentation.

Our third experiment aims to give a comparison between variances fixed and variances updated in the new model. In all the five lines of Figure 3, from left to right are the original image, three membership functions, and hard segmentation, respectively. From the first line to the fourth line are the results with variances fixed. For example, we set $\sigma_{i}^{2}=0.005$ for all $(1 \leq i \leq 3)$ in the first line, and we set $\sigma_{i}^{2}=0.010$ for all $(1 \leq i \leq 3)$ in the second line. However, the last line is the result where variances are updated, and we obtained the final variances for the three phases, which are $\sigma_{1}=0.0069$, $\sigma_{2}=0.0193$, and $\sigma_{3}=0.0135$, respectively. Obviously, the last row gives the best result. This experiment shows that updating variances are better than fixing variances and assuming all of them are equal. Since Shen's model is a special case when all variances are fixed and the same, this experiment shows that the proposed model outperforms Shen's model.

Finally, we test our model using real images. In Figure 4, the liver is not very clear due to the existence of bias. Using Shen's model leads to a wrong result where a big part of the liver was incorrectly classified to background as shown in the first line. This can be easily seen from the hard segmentation. However, using the proposed model can get much better result as shown in the second line. This is because the fitting term in the model contains bias, as well as variance. By calculating the variances of the three phases, they are $0.013,0.011$, and 0.002 , respectively. This fact also proves that it is reasonable to assume that different phases may have different variances as in our model.

As we mentioned at the beginning of the paper, one of the most important application of soft segmentation is partial volume segmentation of MRI brain images. Figure 5 gives a comparison in MRI brain image soft segmentation. There is a big difference between the soft segmentations (the membership functions). By using MAP-AFCM model, most 

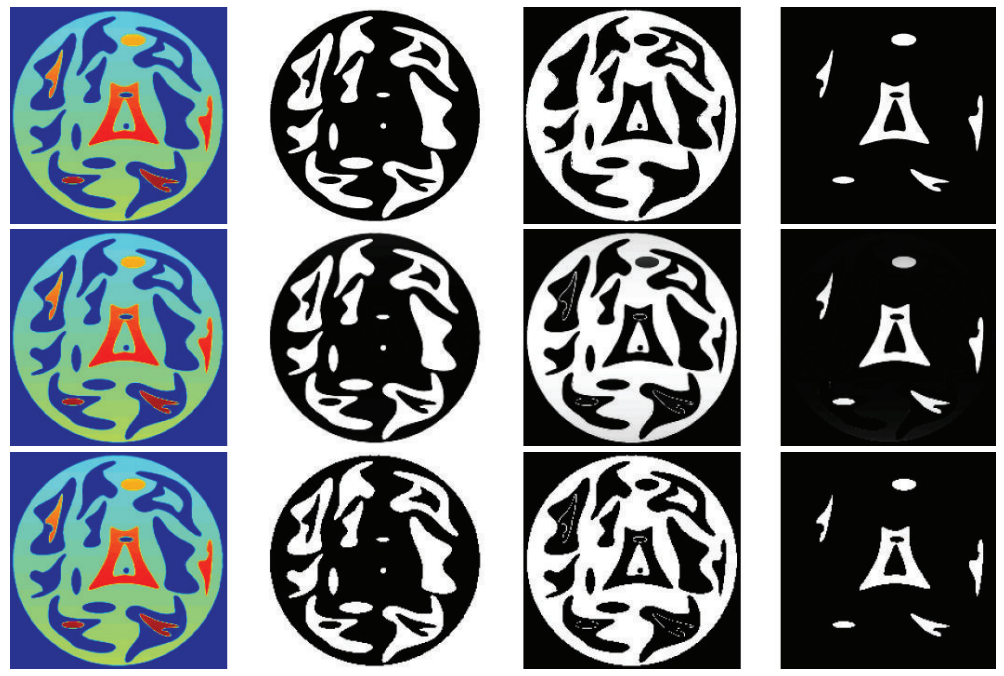

FIGURE 2: Experiment 3: robustness to bias. In the first line from left to right are original image and three ground truth membership functions. In the second line and the third line, from left to right are original image and soft segmentations (three membership functions). The second row is the soft segmentation using Shen's model, and the third line is the soft segmentation using the proposed model.
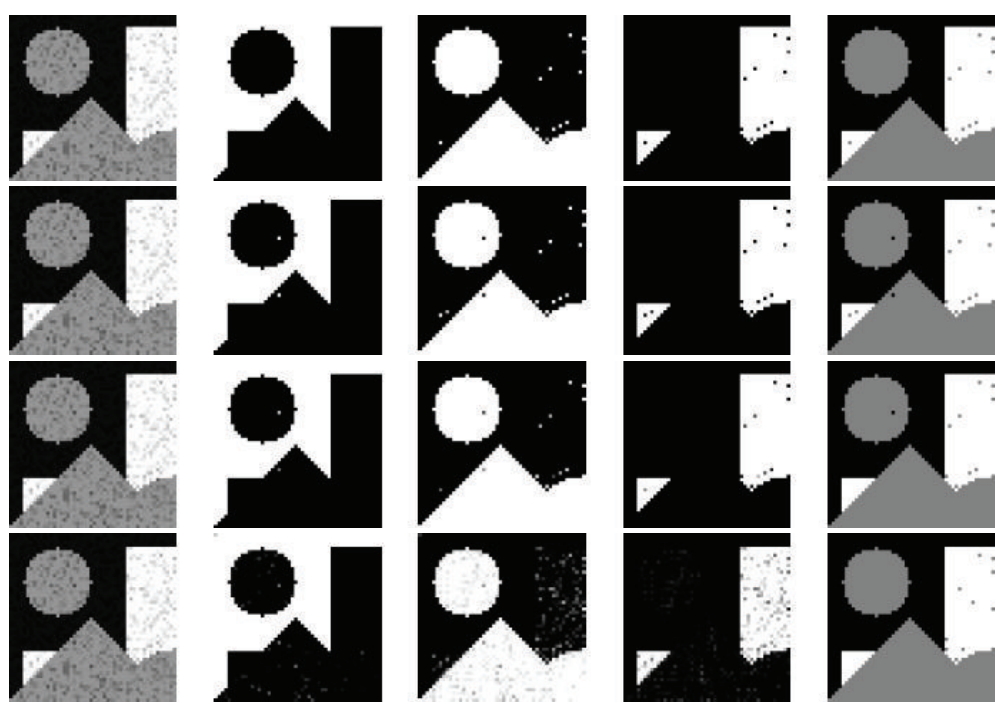

FIGURE 3: Comparison between variances fixed and updated. In every row, the first three graphs are membership functions, and the forth is the hard segmentation after thresholding. Row 1: result with $\sigma=0.005$, Row 2: result with $\sigma=0.010$, Row 3: result with $\sigma=0.015$, Row 4: result with $\sigma=0.020$, and Row 5: result with $\sigma$ updated.
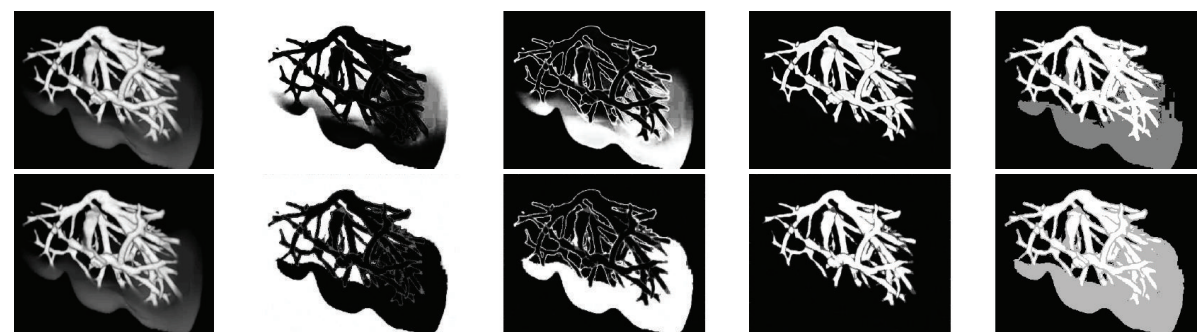

FIgURE 4: MRI liver segmentation. The first line: segmentation using Shen's model. The second line: segmentation using the proposed model. From left to right: original image, three membership functions, and hard segmentations, respectively. 

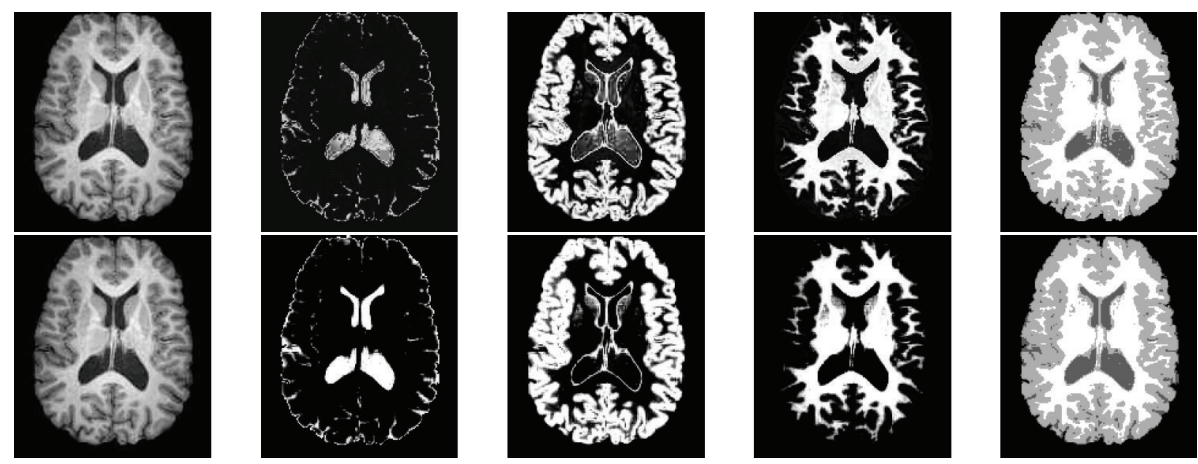

FIGURE 5: MRI brain image segmentations. The first line: segmentation using Shen's model. The second line: segmentation using the proposed model. From left to right: original image, three membership functions (white matter, gray matter, and CSF (cerebrospinal fluid)), and hard segmentations, respectively.
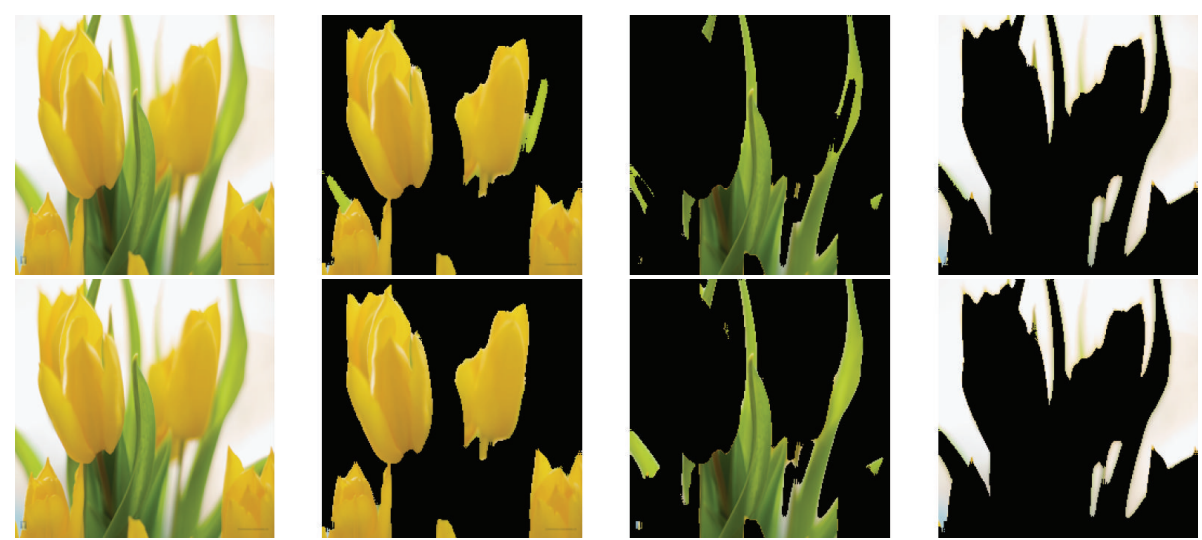

Figure 6: Natural image segmentation after thresholding. From left to right: original image and three phases of hard segmentations. Line 1: Shen's model. Line 2: proposed model.
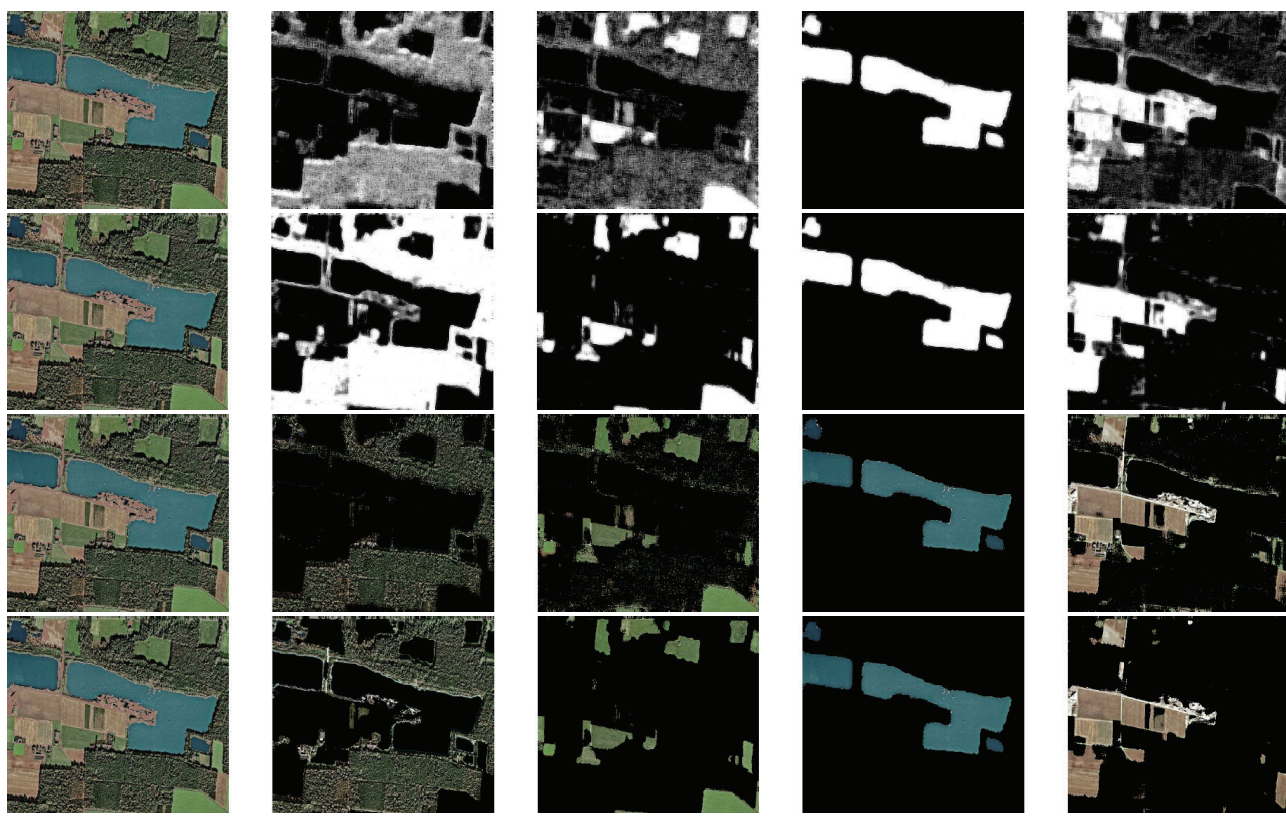

FIGURE 7: Natural image segmentation after thresholding. Line 1: membership functions using Shen's model. Line 2: membership functions using the proposed model. Line 3: hard segmentations using Shen's model. Line 4: hard segmentations using the proposed model. 
pixels are classified to be partial volume, that is, its intensity is neither close to 1 nor close to 0 (in the figure, brightness of intensity means close to 1 , darkness means close to 0 , and intensity between brightness and darkness means partial volume). However, this is not true because it is well known that partial volume of MRI brain image should appear mostly often at the boundary of different tissues. Comparatively, using the proposed model can get more reasonable results, where the partial volume only appears at the boundary of different tissues.

We also present some natural images for comparison. In Figure 6, the left image is the original image, and the middle one and the right one are hard segmentations after thresholding using Shen's model and the proposed model, respectively. In Figure 7, the first column is the original image. We present all membership functions and hard segmentations for readers to compare. For all three examples, the results using our model are all better than using Shen's model.

\section{Conclusion}

In this paper, we proposed a stochastic variational model for multiphase soft segmentation based on Gaussian mixture. Compared with previously associated models, the proposed model is more robust to noise and bias. For implementation, we developed a bi-direction projected PDHG algorithm, which is easy to carry out. Several experiments are presented to demonstrate the efficiency of our new model.

\section{References}

[1] X. Bresson, S. Esedoglu, P. Vandergheynst, J.-P. Thiran, and S. Osher, "Fast global minimization of the active contour/snake model," Journal of Mathematical Imaging and Vision, vol. 28, no. 2, pp. 151-167, 2007.

[2] X. Bresson and T. F. Chan, "Non-local unsupervised variational image segmentation models," UCLA CAM Report, 2008, http://www.math.ucla.edu/.

[3] V. Caselles, R. Kimmel, and G. Sapiro, "Geodesic active contours," International Journal of Computer Vision, vol. 22, no. 1, pp. 61-79, 1997.

[4] T. F. Chan and L. A. Vese, "Active contours without edges," IEEE Transactions on Image Processing, vol. 10, no. 2, pp. 266-277, 2001.

[5] T. F. Chan, S. Esedoglu, and M. Nikolova, "Algorithms for finding global minimizers of image segmentation and denoising models," SIAM Journal on Applied Mathematics, vol. 66, no. 5, pp. 1632-1648, 2006.

[6] D. Mumford and J. Shah, "Optimal approximations by piecewise smooth functions and associated variational problems," Communications on Pure and Applied Mathematics, vol. 42, no. 5, pp. 577-685, 1989.

[7] N. Paragios and R. Deriche, "Geodesic active regions and level set methods for supervised texture segmentation," International Journal of Computer Vision, vol. 46, no. 3, pp. 223-247, 2002.

[8] S. C. Zhu, "Region competition: unifying snakes, region growing, and bayes/mdl for multiband image segmentation," IEEE Transactions on Pattern Analysis and Machine Intelligence, vol. 18, no. 9, pp. 884-900, 1996.
[9] S. Osher and J. A. Sethian, "Fronts propagating with curvaturedependent speed: algorithms based on Hamilton-Jacobi formulations," Journal of Computational Physics, vol. 79, no. 1, pp. 1249, 1988.

[10] G. Chung and L. A. Vese, "Energy minimization based segmentation and denoising using a multilayer level set approach," Energy Minimization Methods in Computer Vision and Pattern Recognition, vol. 3757, pp. 439-455, 2005.

[11] J. Lie, M. Lysaker, and X. C. Tai, "A variant of the level set method and applications to image segmentation," Mathematics of Computation, vol. 75, no. 255, pp. 1155-1174, 2006.

[12] L. A. Vese and T. F. Chan, "A multiphase level set framework for image segmentation using the Mumford and Shah model," International Journal of Computer Vision, vol. 50, no. 3, pp. 271293, 2002.

[13] H.-K. Zhao, T. Chan, B. Merriman, and S. Osher, "A variational level set approach to multiphase motion," Journal of Computational Physics, vol. 127, no. 1, pp. 179-195, 1996.

[14] T. Pock, T. Schoenemann, G. Graber, H. Bischof, and D. Cremers, "A convex formulation of continuous multi-label problems," in Proceedings of the European Conference on Computer Vision (ECCV'08), Marseille, France, October 2008.

[15] E. S. Brown, T. F. Chan, and X. Bresson, "Convex formulation and exact global solutions for multi-phase piecewise constant Mumford-Shah image segmentation," UCLA CAM Report cam09-66, 2009.

[16] E. Bae, J. Yuan, and X.-C. Tai, "Global minimization for continuous multiphase partitioning problems using a dual approach," UCLA CAM Report, 2009, http://www.math.ucla.edu/.

[17] S. Chen and D. Zhang, "Robust image segmentation using FCM with spatial constraints based on new kernel-induced distance measure," IEEE Transactions on Systems, Man, and Cybernetics B, vol. 34, no. 4, pp. 1907-1916, 2004.

[18] X. Li, L. Li, H. Lu, and Z. Liang, "Partial volume segmentation of brain magnetic resonance images based on maximum a posteriori probability," Medical Physics, vol. 32, no. 7, pp. 2337$2345,2005$.

[19] B. Mory and R. Ardon, "Fuzzy region competition: a convex two-phase segmentation framework," in Proceedings of the International Conference on Scale-Space and Variational Methods in Computer Vision, pp. 214-226, 2007.

[20] B. Mory, R. Ardon, and J. P. Thiran, "Variational segmentation using fuzzy region competition and local non-parametric probability density functions," in Proceedings of the IEEE 11th International Conference on Computer Vision (ICCV '07), pp. 18, October 2007.

[21] D. L. Pham and J. L. Prince, "An adaptive fuzzy C-means algorithm for image segmentation in the presence of intensity inhomogeneities," Pattern Recognition Letters, vol. 20, no. 1, pp. 57-68, 1999.

[22] J. Shen, "A stochastic-variational model for soft Mumford-Shah segmentation," International Journal of Biomedical Imaging, vol. 2006, Article ID 92329, 14 pages, 2006.

[23] J. C. Bezdek, "A convergence theorem for the fuzzy ISODATA clustering algorithm," IEEE Transactions on Pattern Analysis and Machine Intelligence, vol. 2, no. 1, pp. 1-8, 1980.

[24] J. C. Dunn, "A fuzzy relative of the ISODATA process and its use in detecting compact wellseparated clusters," Jounal of Cybernetics, vol. 3, no. 3, pp. 32-57, 1973.

[25] F. Chen, Y. Chen, and H. D. Tagare, "An extension of sinesinc model based on logarithm of likelihood," in Proceedings 
of the International Conference on Image Processing, Computer Vision, and Pattern Recognition (IPCV '08), pp. 222-227, July 2008.

[26] A. Dempster, N. Laird, and D. Rubin, "Maximum likelihood from incomplete data via the EM algorithm," Journal Royal Statistical Society B, vol. 39, no. 1, pp. 1-8, 1977.

[27] M. N. Ahmed, S. M. Yamany, N. Mohamed, A. A. Farag, and T. Moriarty, "A modified fuzzy C-means algorithm for bias field estimation and segmentation of MRI data," IEEE Transactions on Medical Imaging, vol. 21, no. 3, pp. 193-199, 2002.

[28] C. Li, R. Huang, Z. Ding, C. Gatenby, D. Metaxas, and J. Gore, "A variational level set approach to segmentation and bias correction of images with intensity inhomogeneity," Medical Image Computing and Computer Assisted Intervention, vol. 11, part 2, pp. 1083-1091, 2008.

[29] W. M. Wells III, W. E. L. Crimson, R. Kikinis, and F. A. Jolesz, "Adaptive segmentation of mri data," IEEE Transactions on Medical Imaging, vol. 15, no. 4, pp. 429-442, 1996.

[30] Y. Boykov and G. Funka-Lea, "Graph cuts and efficient N-D image segmentation," International Journal of Computer Vision, vol. 70, no. 2, pp. 109-131, 2006.

[31] J.-B. Hiriart-Urruty and C. Lemarechal, "Convex analysis and minimization algorithms," in Grundlehren der Mathematischen Wissenschaften, pp. 305-306, Springer, New York, NY, USA, 1993.

[32] A. Chambolle, "An algorithm for total variation minimization and applications," Journal of Mathematical Imaging and Vision, vol. 20, no. 1-2, pp. 89-97, 2004.

[33] M. Zhu and T. Chan, "An efficient primal-dual hybrid gradient algorithm for total variation image restoration," CAM Report cam08-34, 2008.

[34] E. S. Brown, T. F. Chan, and X. Bresson, "Convex relaxation method for a class of vector-valued minimization problems with applications to Mumford-Shah segmentation," UCLA CAM Report cam10-43, 2010.

[35] E. Esser, X. Zhang, and T. Chan, "A general framework for a class of first order primal-dual algorithms for TV minimization," Cam Report cam09-67, 2009.

[36] T. Pock, D. Cremers, H. Bischof, and A. Chambolle, "An algorithm for minimizing the Mumford-Shah functional," in Proceedings of the 12th International Conference on Computer Vision (ICCV'09), pp. 1133-1140, Kyoto, Japan, October 2009.

[37] T. Pock, D. Cremers, H. Bischof, and A. Chambolle, "An algorithm for minimizing the Mumford-Shah functional," in Proceedings of the 12th International Conference on Computer Vision (ICCV '09), pp. 1133-1140, October 2009. 

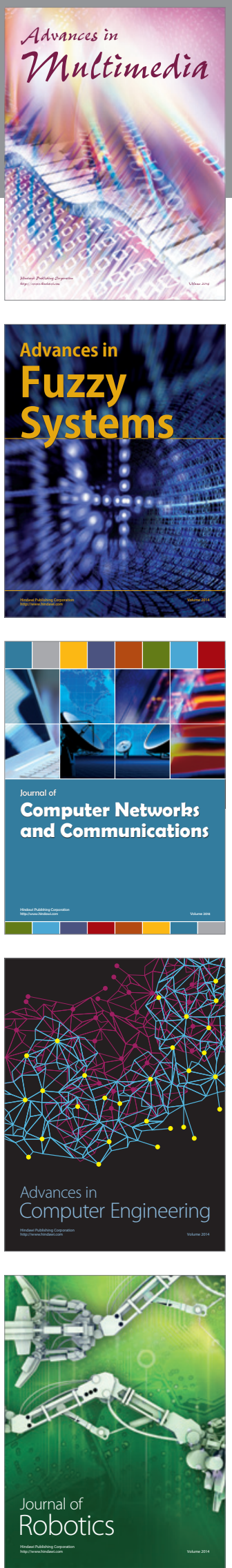

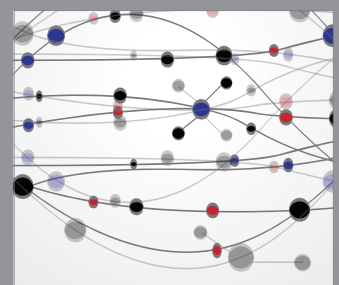

The Scientific World Journal
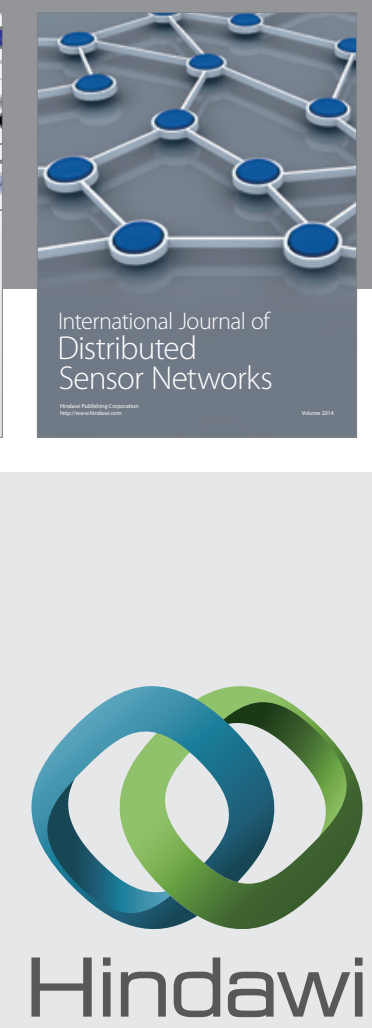

Submit your manuscripts at

http://www.hindawi.com
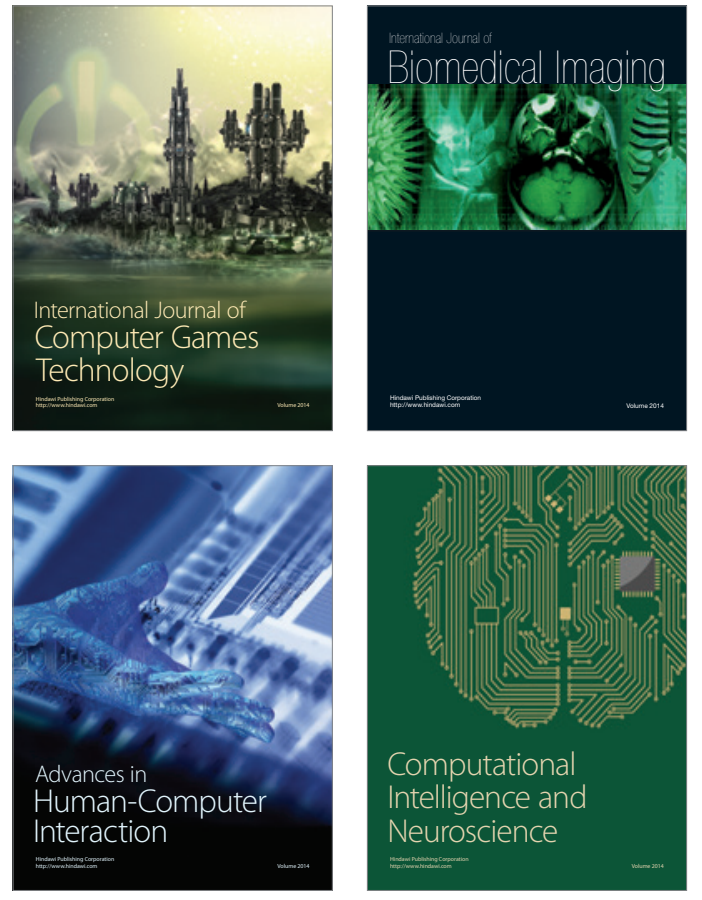
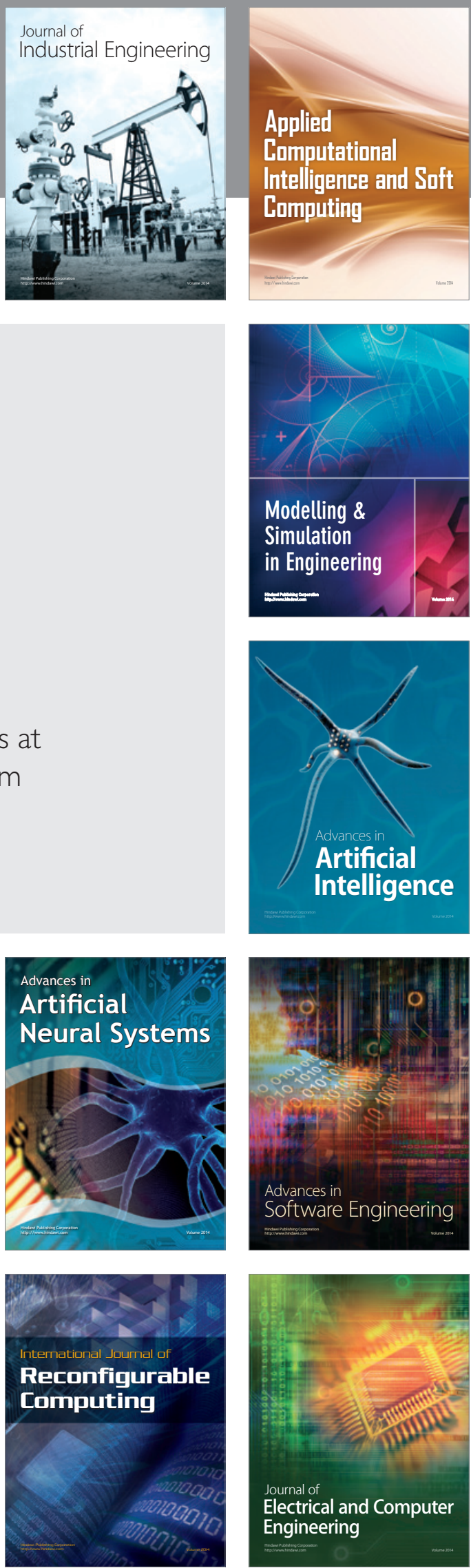